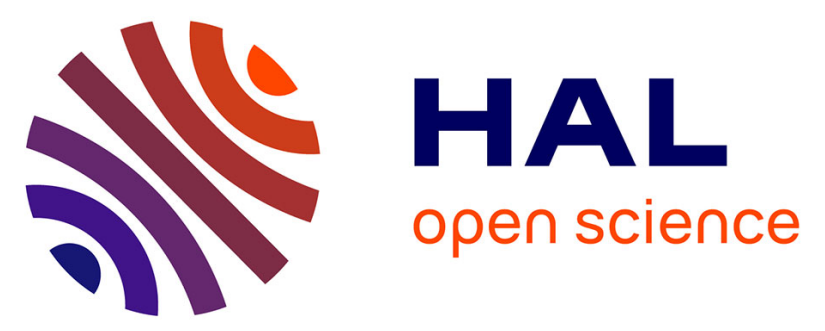

\title{
imzML: Imaging Mass Spectrometry Markup Language: A Common Data Format for Mass Spectrometry Imaging
}

Andreas Römpp, Thorsten Schramm, Alfons Hester, Ivo Klinkert, Jean-Pierre Both, Ron M. A. Heeren, Markus Stoeckli, Bernhard Spengler

\section{To cite this version:}

Andreas Römpp, Thorsten Schramm, Alfons Hester, Ivo Klinkert, Jean-Pierre Both, et al.. imzML: Imaging Mass Spectrometry Markup Language: A Common Data Format for Mass Spectrometry Imaging. Methods in Molecular Biology, 2011, 696, pp.205-224. 10.1007/978-1-60761-987-1_12 . cea-03086182

\section{HAL Id: cea-03086182 https://hal-cea.archives-ouvertes.fr/cea-03086182}

Submitted on 22 Dec 2020

HAL is a multi-disciplinary open access archive for the deposit and dissemination of scientific research documents, whether they are published or not. The documents may come from teaching and research institutions in France or abroad, or from public or private research centers.
L'archive ouverte pluridisciplinaire HAL, est destinée au dépôt et à la diffusion de documents scientifiques de niveau recherche, publiés ou non, émanant des établissements d'enseignement et de recherche français ou étrangers, des laboratoires publics ou privés. 


\title{
imzML: Imaging Mass Spectrometry Markup Language: A Common Data Format for Mass Spectrometry Imaging
}

\author{
Andreas Römpp, Thorsten Schramm, Alfons Hester, Ivo Klinkert, \\ Jean-Pierre Both, Ron M.A. Heeren, Markus Stöckli, \\ and Bernhard Spengler
}

\begin{abstract}
Imaging mass spectrometry is the method of scanning a sample of interest and generating an "image" of the intensity distribution of a specific analyte. The data sets consist of a large number of mass spectra which are usually acquired with identical settings. Existing data formats are not sufficient to describe an MS imaging experiment completely. The data format imzML was developed to allow the flexible and efficient exchange of MS imaging data between different instruments and data analysis software.

For this purpose, the MS imaging data is divided in two separate files. The mass spectral data is stored in a binary file to ensure efficient storage. All metadata (e.g., instrumental parameters, sample details) are stored in an XML file which is based on the standard data format mzML developed by HUPO-PSI. The original mzML controlled vocabulary was extended to include specific parameters of imaging mass spectrometry (such as $\mathrm{x} / \mathrm{y}$ position and spatial resolution). The two files (XML and binary) are connected by offset values in the XML file and are unambiguously linked by a universally unique identifier. The resulting datasets are comparable in size to the raw data and the separate metadata file allows flexible handling of large datasets.

Several imaging MS software tools already support imzML. This allows choosing from a (growing) number of processing tools. One is no longer limited to proprietary software, but is able to use the processing software which is best suited for a specific question or application. On the other hand, measurements from different instruments can be compared within one software application using identical settings for data processing. All necessary information for evaluating and implementing imzML can be found at http://www.imzML.org.
\end{abstract}

\section{Introduction}

Imaging mass spectrometry is the method of scanning a sample of interest and generating an "image" of the intensity distribution of a specific analyte. The principle of this method is shown 


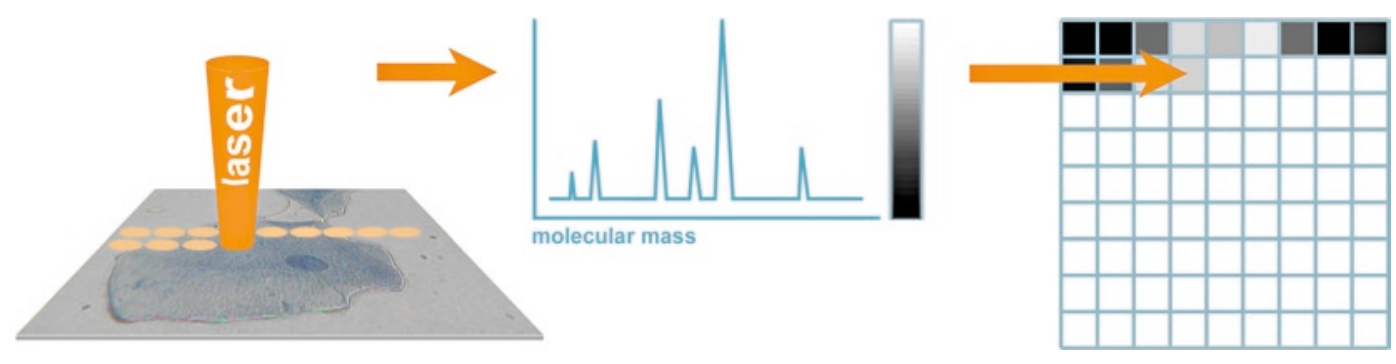

Fig. 1. Schematic process of scanning microprobe MALDI-MS. A desorption laser scans the surface of the target (e.g., a cell or tissue). The intensity of a selected peak in the resulting mass spectrum is transformed into a pixel of a grayscale image.

in Fig. 1. The application of MS imaging is rapidly growing with a constantly increasing number of different instrumental systems and software tools. An overview of methods and applications of mass spectrometry imaging has been recently published (1). This method results in a large number of spectra which are typically acquired with identical measurement parameters. The data format described in this chapter was developed within the EU-funded project COMPUTIS (2). The goal of this project was to develop new and improved technologies for molecular imaging mass spectrometry. An important task was the comparison of images generated by diverse types of mass spectrometers. Therefore a standard format for the exchange of MS imaging data was needed. Both the DICOM standard for in-vivo imaging data (3) and the mzML standard (4) by HUPO-PSI $(5,6)$ are not able to completely represent an imaging MS experiment. Therefore a standardized data format was developed to simplify the exchange of imaging MS data between different instrument and data analysis software. The following institutions were involved in the development of imzML: Justus Liebig University (JLU), Giessen, Germany; FOM Institute for Atomic and Molecular Physics (FOM), Amsterdam, The Netherlands; Commissariat à l'Énergie Atomique (CEA), Saclay, France; and Novartis Institutes for BioMedical Research (Novartis), Basel, Switzerland.

Several data formats for MS imaging utilize two separate files: a small file (ini or XML) for the metadata and a larger (binary) file for the mass spectral data (e.g., Biomap (7) and internal data formats at FOM and JLU). This structure proved to be very useful for flexible and fast handling of the imaging MS data and it was decided to follow this approach for the new data format. In order to keep as close as possible to existing formats, we decided that the (small) metadata file should be based on the mass spectrometry standard mzML developed by HUPO-PSI (8). A more detailed discussion on why mzML was not fully implemented and about the relation between the two data formats (mzML and imzML) is found in Note 1. A new controlled vocabulary was 
compiled for imzML to include parameters that are specific for imaging experiments (see Subheading 2.1.2). All relevant information about imzML including specifications and example files can be found at http://www.imzML.org.

The following section describes the design philosophy of imzML. The data structure is discussed in more detail in Subheading 2. Properties and possibilities of imzML files are discussed in Subheading 4. Available software applications including an example for a file converter are presented in Subheading 5 .

\section{2. imzML Data}

\section{Format}

\subsection{Data Structure}

The fundamental goal while developing imzML was to design a data format for the efficient exchange of mass spectrometry imaging data. At the same time, the format should be easily interchangeable with mzML.

The main goals can be summarized as

1. Ensure complete description of imaging MS experiments

2. Minimize file size

3. Ensure fast and flexible data handling

4. Keep the (XML part of) imzML as close as possible to mzML

imzML consists of two separate files: one for the metadata and one for the mass spectral data. The metadata is saved in an XML file (*.imzML). The mass spectral data is saved in a binary file (*.ibd). A schematic representation of the imzML file structure is shown in Fig. 2. The connection between the two files is made via

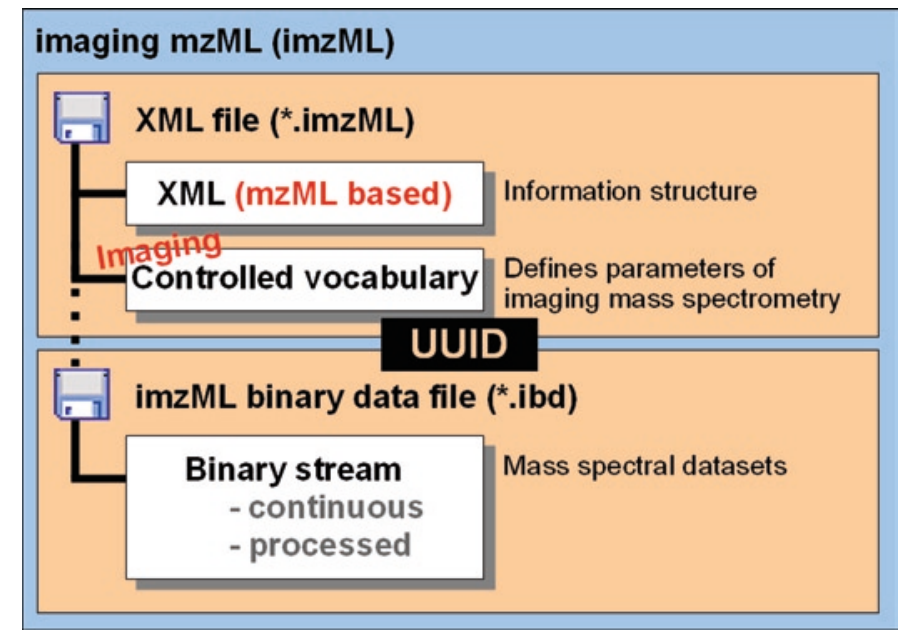

Fig. 2. Data structure of imzML. 
2.1.2. Controlled Vocabulary links in the XML file which contain the offset positions of the mass spectra in the binary file. It is important to keep in mind that the information is only valid if both files are available. Therefore the user should be very careful when copying or moving those files; inaccurate file handling can result in data loss. It is recommended to keep both files in the same folder and to use the same names for the .imzML part and the ibd part.

Corresponding XML and binary files contain the same universally unique identifier (UUID) (9) in order to link them unequivocally. The UUID is a controlled vocabulary entry in the $<$ fileContent $>$ tag of the XML file and is also stored at the beginning of the binary file. Comparing both UUIDs allows finding out if the two files belong to the same measurement/data set. More details on the implementation of UUIDs are discussed in Note 2 .

The XML file holds the metadata of a MS imaging experiment which is described by the mzML-based XML structure and the controlled vocabulary. The XML model of imzML is the same as for mzML (see the mzML version 1.1.0 documentation for further details) (8). The controlled vocabulary was extended in order to include additional parameters which are needed to describe an MS imaging experiment (see Subheading 2.1.2). Most of the changes in the XML part are related to cvParam mapping rules for the newly introduced parameters of the imaging controlled vocabulary. One of the most important changes compared to mzML is the function of the < binary > element which not contain base64-encoded binary data anymore. It stays empty, which is compatible to mzML 1.1.0. This results in predefined values for "encoded length" and "array length" of zero in the parent tags $<$ spectrum $>$ and < binaryDataArray $>$. The XML part of imzML passes mzML validators without errors (only warning messages for unknown cv entries are displayed). An example of XML code is given in Fig. 3. Modifications (compared to mzML) are printed bold and will be discussed in more detail in the following section.

The controlled vocabulary is used to unequivocally describe the information in the XML file. The additional imzML CV terms are stored in an open biomedical ontology $((10,11))$ - the imagingMS.obo file (12). They complement the mass spectrometry parameters of the MS controlled vocabulary provided by HUPOPSI (13) in order to allow a complete description of MS imaging experiments. An overview of CV entries concerning imaging-specific parameters and image properties is given in Table 1. These parameters include information about the image itself and acquisition parameters. For example: How many pixels does the image contain in the $\mathrm{x}$ and $\mathrm{y}$ dimension? Which position in 


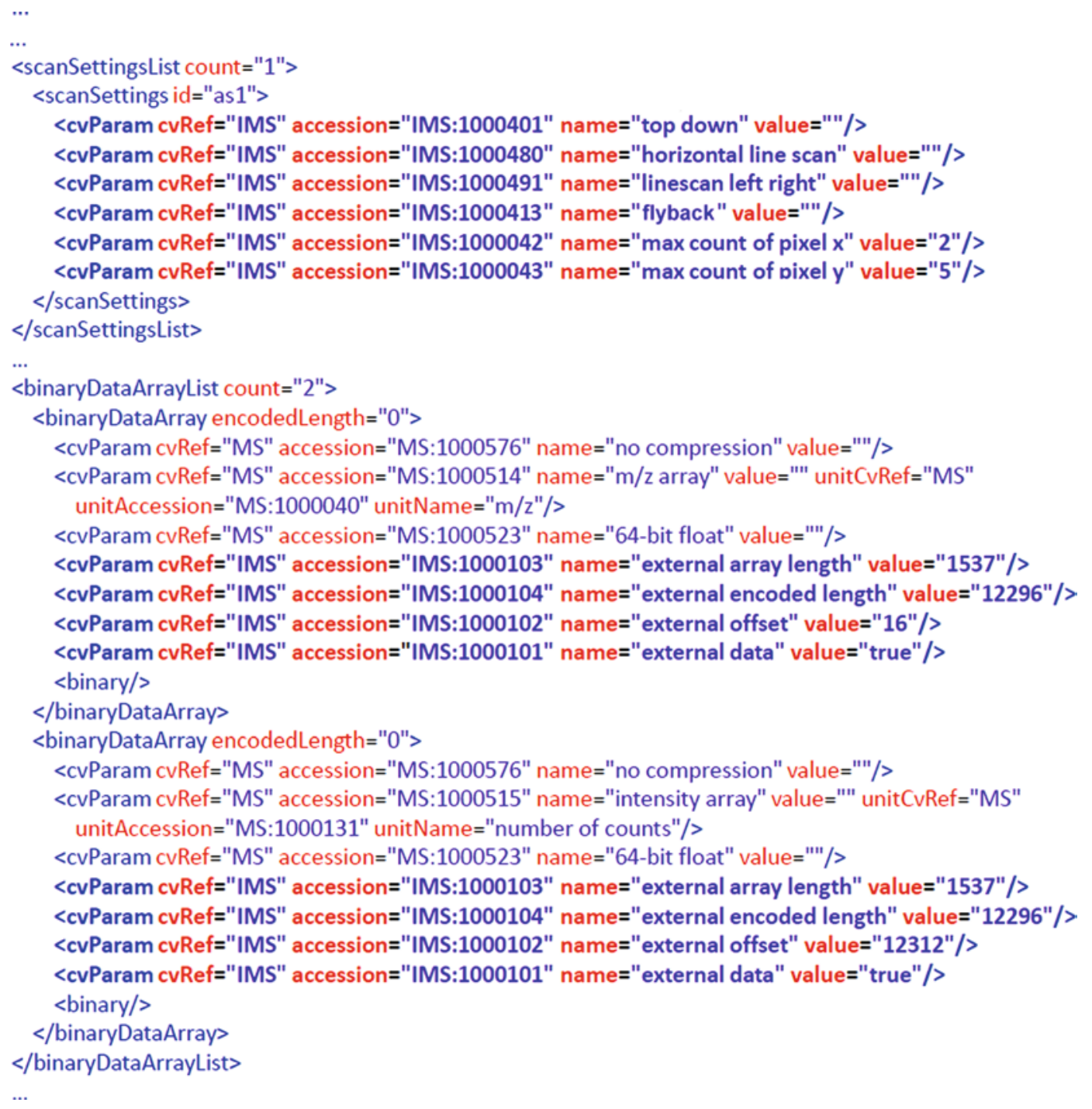

Fig. 3. XML code new CV entries are printed bold.

the image belongs to which spectrum? In which pattern was the image scanned? Which Matrix was used in which concentration? It is also necessary to give information about the instrumentation used. In addition to the mass spectrometer, the ablation laser type and parameters have to be documented such as wavelength, energy, and impact angle. The sample stage also plays an essential role when generating an image: step size and position accuracy. The parameters concerning the scanning process are explained in more detail in Subheading "Image Orientation".

Additional entries were included, which are necessary for handling the external binary file. Four parameters were introduced 


\section{Table 1 \\ Additional parameters of the controlled vocabulary}

\begin{tabular}{|c|c|}
\hline $\begin{array}{l}\text { ibd file: } \\
\text { External binary uri }\end{array}$ & Location as an URI where to find the ibd file. \\
\hline $\begin{array}{l}\text { ibd checksum: } \\
\text { ibd MD5 }\end{array}$ & $\begin{array}{l}\text { MD5 (Message-Digest algorithm } 5 \text { ) is a cryptographic hash } \\
\text { function with a 128-bit hash value used to check the integrity of } \\
\text { files. } \\
\text { SHA-1 (Secure Hash Algorithm-1) is a cryptographic hash function } \\
\text { designed by the National Security Agency (NSA) and published } \\
\text { by the NIST as a U. S. government standard. It is also used to } \\
\text { verify file integrity. }\end{array}$ \\
\hline $\begin{array}{l}\text { ibd binary type: } \\
\text { Continuous }\end{array}$ & $\begin{array}{l}\text { Way of saving spectra in an imzML binary data file (ibd). The } \mathrm{m} / \mathrm{z} \\
\text { values for all spectra are saved at the beginning of the ibd file. } \\
\text { Then the spectral values follow. }\end{array}$ \\
\hline Processed & $\begin{array}{l}\text { Way of saving spectra in an imzML binary data file (ibd). Every } \\
\text { spectrum is saved with its own } \mathrm{m} / \mathrm{z} \text { and intensity values. }\end{array}$ \\
\hline $\begin{array}{l}\text { ibd identification: } \\
\text { Universally unique identifier }\end{array}$ & $\begin{array}{l}\text { Universally unique identifier is unique throughout the world and } \\
\text { allows to doubtlessly identify the ibd file. }\end{array}$ \\
\hline $\begin{array}{l}\text { ibd offset handle: } \\
\text { External array length } \\
\text { External data } \\
\text { External encoded length } \\
\text { External offset }\end{array}$ & $\begin{array}{l}\text { Describes how many fields the external data array contains. } \\
\text { Shows that there is no data in the <binary> section of the file but } \\
\text { saved in an external file. } \\
\text { Describes the length of the written data stream in byte. } \\
\text { The position in byte where the data of the data array of a mass } \\
\text { spectrum begins. }\end{array}$ \\
\hline $\begin{array}{l}\text { Image: } \\
\text { Absolute position offset } x \\
\text { Absolute position offset } y \\
\text { Max count of pixels } x \\
\text { Max count of pixels } y \\
\text { Max dimension } x \\
\text { Max dimension } y \\
\text { Pixel size } x \\
\text { Pixel size } y \\
\text { Image shape }\end{array}$ & $\begin{array}{l}\text { Describes the position at the } x \text {-axis of the upper left point of the } \\
\text { image on the target. } \\
\text { Describes the position at the } y \text {-axis of the upper left point of the } \\
\text { image on the target. } \\
\text { Maximum number of pixels of the } x \text {-axis of the image. } \\
\text { Maximum number of pixels of the y-axis of the image. } \\
\text { Maximum length of the image in } x \text {-axis. } \\
\text { Maximum length of the image in y-axis. } \\
\text { Describes the length in } x \text {-direction of the pixels. } \\
\text { Describes the length in y-direction of the pixels. } \\
\text { Describes the shape of the image. }\end{array}$ \\
\hline $\begin{array}{l}\text { Laser shot mode: } \\
\text { Pixel mode } \\
\text { Raster mode } \\
\text { Stigmatic mode }\end{array}$ & $\begin{array}{l}\text { The laser keeps the position while firing at the same spot one or } \\
\text { several times. } \\
\text { The laser is moved while continuously firing at the sample. } \\
\text { The laser is moved around one point firing until moved to the next } \\
\text { position (pixel). }\end{array}$ \\
\hline
\end{tabular}




\section{Table 1 \\ (continued)}

\begin{tabular}{|c|c|}
\hline \multicolumn{2}{|l|}{ Spectrum position: } \\
\hline Position $\mathrm{x}$ & $\begin{array}{l}\text { Attribute to describe the position of a spectrum in the direction of } \\
\text { the } x \text {-axis in the image. }\end{array}$ \\
\hline Position y & $\begin{array}{l}\text { Attribute to describe the position of a spectrum in the direction of } \\
\text { the y-axis in the image. }\end{array}$ \\
\hline Position z & $\begin{array}{l}\text { Attribute to describe the position of a spectrum in the direction of } \\
\text { the } \mathrm{z} \text {-axis in the image. }\end{array}$ \\
\hline Subimage position $\mathrm{x}$ & $\begin{array}{l}\text { Describes the position of a subimage in the direction of the } x \text {-axis of } \\
\text { the complete image. }\end{array}$ \\
\hline Subimage position y & $\begin{array}{l}\text { Describes the position of a subimage in the direction of the } y \text {-axis of } \\
\text { the complete image. }\end{array}$ \\
\hline Subimage position $\mathrm{z}$ & $\begin{array}{l}\text { Describes the position of a subimage in the direction of the } \mathrm{z} \text {-axis of } \\
\text { the complete image. }\end{array}$ \\
\hline \multicolumn{2}{|l|}{ Sample stage: } \\
\hline Target material & Describes the material the target is made of. \\
\hline Linescan sequence: & \\
\hline Bottom up & $\begin{array}{l}\text { The starting point is at the bottom of the sample and the sequence } \\
\text { of the linescans is in up direction (parallel to the y-axis). }\end{array}$ \\
\hline Top down & $\begin{array}{l}\text { The starting point is at the top of the sample and the sequence of } \\
\text { the linescans is in bottom direction (parallel to the y-axis). }\end{array}$ \\
\hline Left right & $\begin{array}{l}\text { The starting point is at the left of the sample and the sequence of } \\
\text { the linescans is in right direction (parallel to the } \mathrm{x} \text {-axis). }\end{array}$ \\
\hline Right left & $\begin{array}{l}\text { The starting point is at the right of the sample and the sequence of } \\
\text { the linescans is in left direction. (parallel to the } x \text {-axis). }\end{array}$ \\
\hline No direction & $\begin{array}{l}\text { The linescans are performed randomly on the sample without any } \\
\text { sequence. }\end{array}$ \\
\hline
\end{tabular}

Scan pattern:

Meandering

The scanning happens in non-stop way. As soon as the end of the sample is reached, the scanning direction will be switched and the scanning is continued. There is no new positioning necessary.

Flyback

The scanning always happens in the same direction. As soon as the end of the sample is reached, the stage is positioned at the starting edge to begin the next run.

Random access

The scanning points are randomly chosen and do not follow a pattern.

Scan type:

Horizontal linescan

Vertical linescan

Linescan direction:

Linescan bottom up

Linescan left right

Linescan right left

Linescan top down
The scanning line is a horizontal one.

The scanning line is a vertical one.
The starting point is at the bottom of the sample and the scanning happens in up direction (parallel to the y-axis).

The starting point is at the left of the sample and the scanning happens in right direction (parallel to the $\mathrm{x}$-axis).

The starting point is at the right of the sample and the scanning happens in left direction. (parallel to the $\mathrm{x}$-axis).

The starting point is at the top of the sample and the scanning happens in bottom direction (parallel to the $y$-axis). 
2.1.2.1. Image Orientation

2.1.2.2. Scan Pattern into the controlled vocabulary to describe the position and length of the data in the binary file. The "external data" parameter indicates that the mass spectral data is stored in a binary file. The parameter "external offset" holds the information at which byte in the binary file the data of the corresponding array starts. If one adds the value of the "external encoded length" to the value of the "external offset" the result has to be equal to the "external offset" of the following binary data array. The parameter "external encoded length" describes the byte length, which has to be read to obtain all the data of the array completely. The parameter "external array length" indicates the number of values of the array.

A separate checksum for the binary file was added in order to find out if the external file has been manipulated or corrupted. It can be either a SHA-1 (Secure Hash Algorithm) or a MD-5 (Message-Digest Algorithm) hash.

Ensuring the integrity and authenticity of digital data is of growing importance in today's information management systems especially for companies who have to operate in accordance with GLP regulations (14). Therefore imzML contains a mechanism to monitor (intentional or accidental) modifications of the data. This feature is based on asymmetric cryptosystems, also known as public key systems and hash functions. The checksum of the binary file is encrypted with a personal key. A public key is needed in order to verify the integrity of the checksum (and therefore the data). This feature (in its basic version) only requires three additional CV entries in the XML file and can thus be easily added to existing imzML files. This modular setup also allows for changing the used encryption procedure, e.g., when one method turns out not to be secure anymore. The encryption is an additional optional feature. This means the data is still readable without the public key, but authenticity cannot be tested in this case. This will protect data against loss (e.g., when one of the keys is lost) and also ensures readability of the data with (older) software that does not include the encryption feature.

The pixel in the upper left corner of the MS image is defined as position $1 / 1$ (Fig. 4). This way every application should generate images of identical orientation. This particular orientation was chosen because it is used for image acquisition in several MS imaging systems. The information of $\mathrm{x}$ and $\mathrm{y}$ position is part of the CV parameters in the <spectrum $>$ tag of each mass spectrum (see also Fig. 3).

Information about the pattern and sequence in which an image was acquired is not necessary for generating an image from the imzML file (because $\mathrm{x}$ and $\mathrm{y}$ position are specified for each individual spectrum). But these parameters can be very important for 


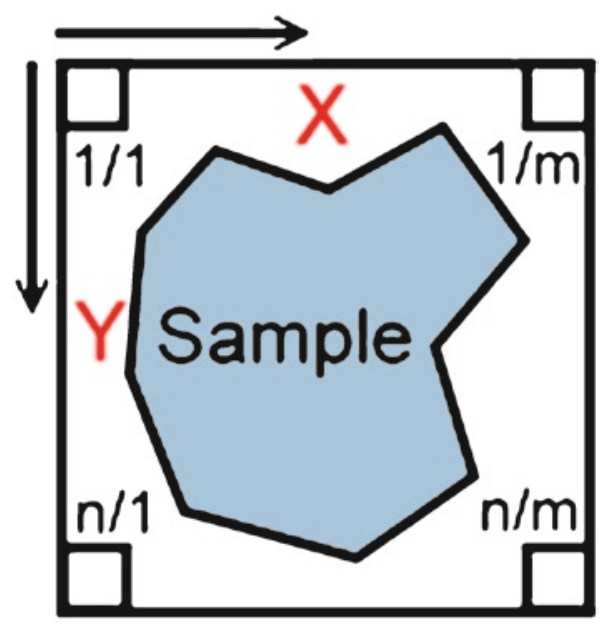

Fig. 4. sample orientation.

Scan pattern: one way

Scan type: horizontal linescan

Linescan direction: left right

Linescan sequence: top down

Scan pattern: one way

Scan type: vertical linescan

Linescan direction: top down

Linescan sequence: right left

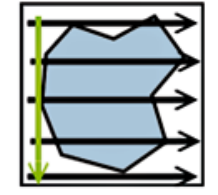

Scan pattern: meandering

Scan type: horizontal linescan

Linescan direction: left right

Linescan sequence: bottom up

Scan pattern: meandering

Scan type: vertical linescan

Linescan direction: top down

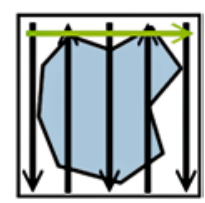

Linescan sequence: left right
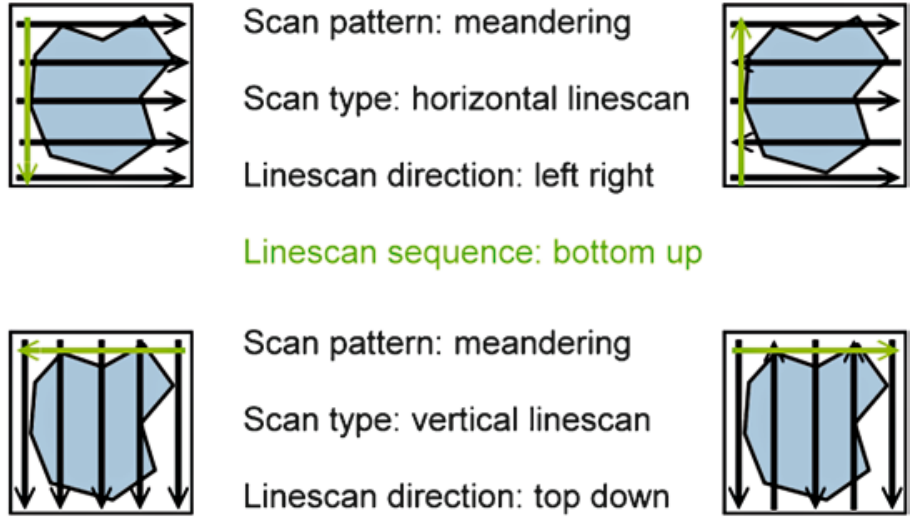

Fig. 5. Examples of scan patterns.

data analysis and interpretation and are therefore part of the imzML controlled vocabulary. The scan process is unambiguously described by four parameters. The different scan parameters are illustrated by examples in Fig. 5. The parameter scan pattern gives information if the sample was scanned in fly-back or meandering mode. Fly-back means that the linescans always occur in the same direction. Meandering indicates that the linescans occur in alternating direction. The scan type defines horizontal or vertical linescans. The linescan direction defines the direction of the (first) linescan(s). The linescan sequence specifies the chronological order of the linescans. 
continuous

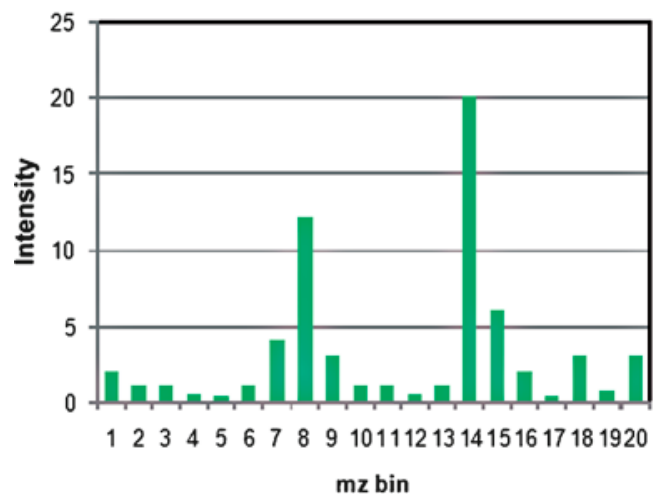

processed

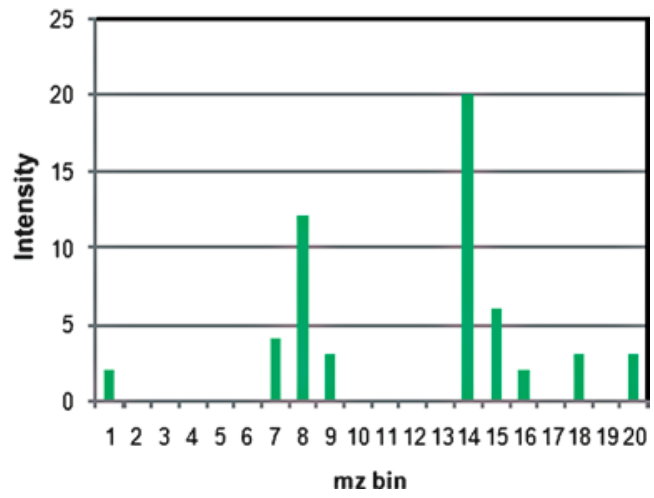

Fig. 6. Data types.

The imaging binary data file (*.ibd) contains the mass spectral data of the MS imaging measurement. The first 16 bytes of the binary file are reserved for the UUID. This identifier is also saved in the corresponding XML file so that a correct assignment of ibd and XML file is possible even if the names of both files are different. In order to ensure efficient storage, two different binary modes are defined: continuous and processed. Schematic examples of these two data types are shown in Fig. 6. "Continuous" means that an intensity value is stored for each $\mathrm{m} / \mathrm{z}$ bin even if there is no signal (resulting in an intensity of zero). As a result, the $\mathrm{m} / \mathrm{z}$ axis is identical for all spectra of one image (if the mass range and bin size is not changed). Therefore it is sufficient to store the $\mathrm{m} / \mathrm{z}$ array only once in the binary file (directly behind the UUID). For each of the spectra only the corresponding intensity values are stored. This structure can reduce the file size significantly (see Subheading 3).

On the other hand mass spectra are often processed before they are stored, e.g., for noise-reduction, peak-picking, deisotoping. This results in discontinuous and non-constant $\mathrm{m} / \mathrm{z}$ arrays. In this case, the $\mathrm{m} / \mathrm{z}$ array has to be stored for each spectrum separately. These data are stored as alternating $\mathrm{m} / \mathrm{z}$ and intensity arrays in the binary file of imzML. The different storage types are illustrated in Fig. 7. More information on choosing the right binary mode is given in Note 3 .

The second parameter influencing the file size is the binary data type, which is used to store the values of the spectra. The imzML binary format allows the storage of values in the following signed integer types: 8 bit, 16 bit, and 32 bit. The values can also be saved in floating point data types (IEEE 754): 32 bit (single precision) or 64 bit (double precision). The byte order of mass spectral data in imzML is little endian (see also Note 2). One value saved in the 32 bit integer type needs four bytes of disk space. The disk space needed by all values of all spectra determines the 
processed

\begin{tabular}{|l|}
\hline mzArray 1 \\
\hline Intensity array $\mathbf{1}$ \\
\hline mzArray 2 \\
\hline Intensity array 2 \\
\hline mzArray 3 \\
\hline Intensity array 3 \\
\hline mzArray 4 \\
\hline Intensity array 4 \\
\hline mzArray 5 \\
\hline Intensity array 5 \\
\hline mzArray 6 \\
\hline Intensity array 6 \\
\hline mzArray 7 \\
\hline Intensity array 7 \\
\hline mzArray 8 \\
\hline Intensity array 8 \\
\hline mzArray 9 \\
\hline Intensity array 9 \\
\hline mzArray 10 \\
\hline Intensity array $\mathbf{1 0}$ \\
\hline
\end{tabular}

continuous

mzArray
Intensity array 1
Intensity array 2
Intensity array 3
Intensity array 4
Intensity array 5
Intensity array 6
Intensity array 7
Intensity array 8
Intensity array 9
Intensity array 10

Fig. 7. Binary data formats.

overall file size. Therefore the choice of the data types for intensity and $\mathrm{m} / \mathrm{z}$ values directly influences the file size of the binary file. The data type is specified for each binary data array separately by CV parameters. More details can be found in Note 4 .

\section{3. imzML File Properties}

The efficient data storage of the imzML format is demonstrated by an example file consisting of 7,000 spectra (Fig. 8). This file represents a measurement on a linear ion trap mass spectrometer (LTQ, Thermo Scientific GmbH, Bremen) (linear ion trap) of 50 by 35 pixel with four spectra (profile mode) acquired for each pixel. The original raw data in the proprietary LTQ format has a file size of $215 \mathrm{MB}$. The imzML files were saved with the following settings: $\mathrm{m} / \mathrm{z}$ values were stored as 32 bit float and intensity values as 32 bit integer. Conversion of this data to an mzML file results in a file size of $577 \mathrm{MB}$. The imzML files are $430 \mathrm{MB}$ and $217 \mathrm{MB}$ for the processed and continuous mode, respectively. The smaller size of the processed imzML file compared to the mzML file is mainly due to base 64 encoding of the mass spectral 


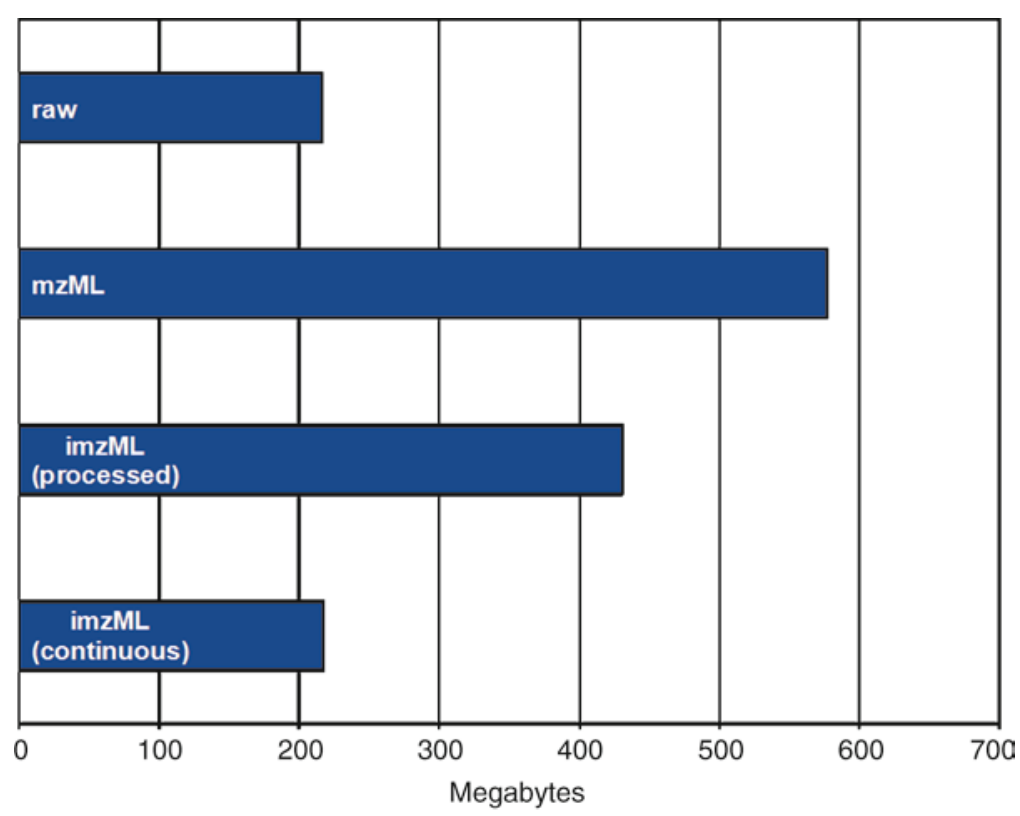

Fig. 8. File size comparison.

data in the mzML file. The even smaller file size of the continuous imzML file is due to the fact that the $\mathrm{m} / \mathrm{z}$ array (which is identical for all mass spectra) is stored only once at the beginning of the binary file (see Subheading 2.1.3 for more details). The continuous imzML file is slightly larger $(2 \mathrm{MB})$ than the original raw file.

In addition to the smaller overall size of the imzML files, the small XML file (several MB) can be used to distribute metadata over the network. This information can be used to select interesting measurements for which the (large) binary files are downloaded selectively (as opposed to downloading the complete dataset for all samples).

\section{Implementation}

imzML is already implemented in a number of software tools (some of them are available on http://www.imzML.org). Several vendors of mass spectrometry imaging instrumentation support the format (e.g., through export filters). Please check the imzML website (http://www.imzML.org) for updated information on supported vendor platforms and available tools.

There are numerous ways to display and analyze MS imaging data and no single software application can combine all features. Therefore it is a big advantage if one can freely choose the most 
appropriate software and is not limited by (proprietary) data formats anymore. Examples of software tools that support imzML to display and analyze MS imaging data are shown in the following. A standard sample (peptide solution on a stainless steel target) was used for a round-robin experiment in order to compare different mass spectrometry imaging systems. The images below show the analysis of this particular measurement on a linear ion trap mass spectrometer with different software tools. The selected ion image of $\mathrm{m} / \mathrm{z} 573$ is shown for each tool in order to verify that the data is read and displayed correctly. The specific advantages of each software are illustrated with selected examples.

Biomap by Novartis (Fig. 9) is one of the most widely used software tools for mass spectrometry imaging. It allows browsing through selected ion images as well as coregistration of images and includes a large number of additional analysis tools.

The Datacube Explorer tool by FOM allows dynamic scrolling through masses in a dataset for fast and easy screening of a

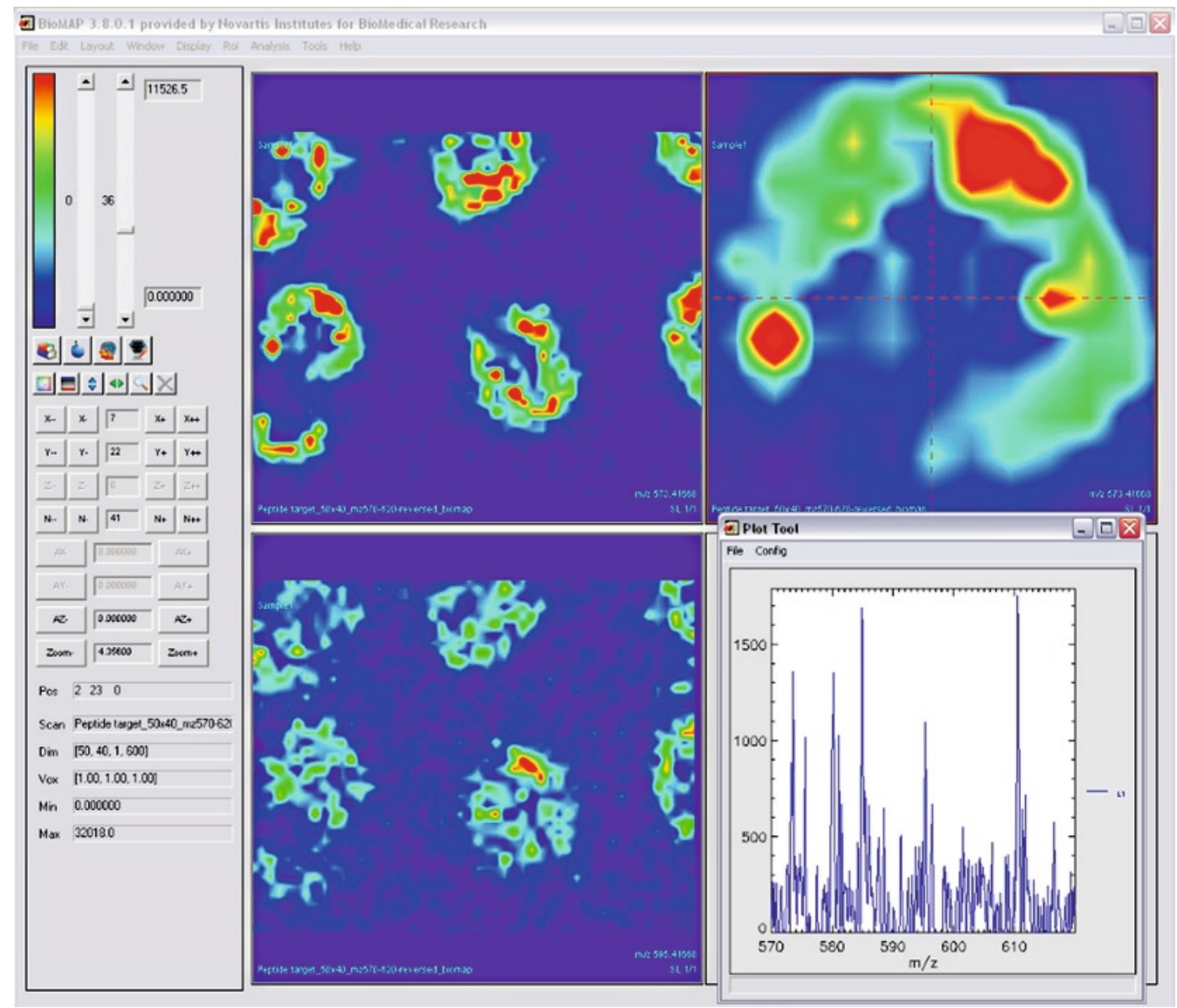

Fig. 9. BioMap (Novartis). 
dataset (Fig. 10). It also allows spectral analysis of regions of interest and contains advanced analysis features such as self-organizing maps for image classification. This tool is available for free on http://www.imzML.org.

The fxSpectViewer by CEA is especially suited for handling very large data files without the need of binning (Fig. 11). It also

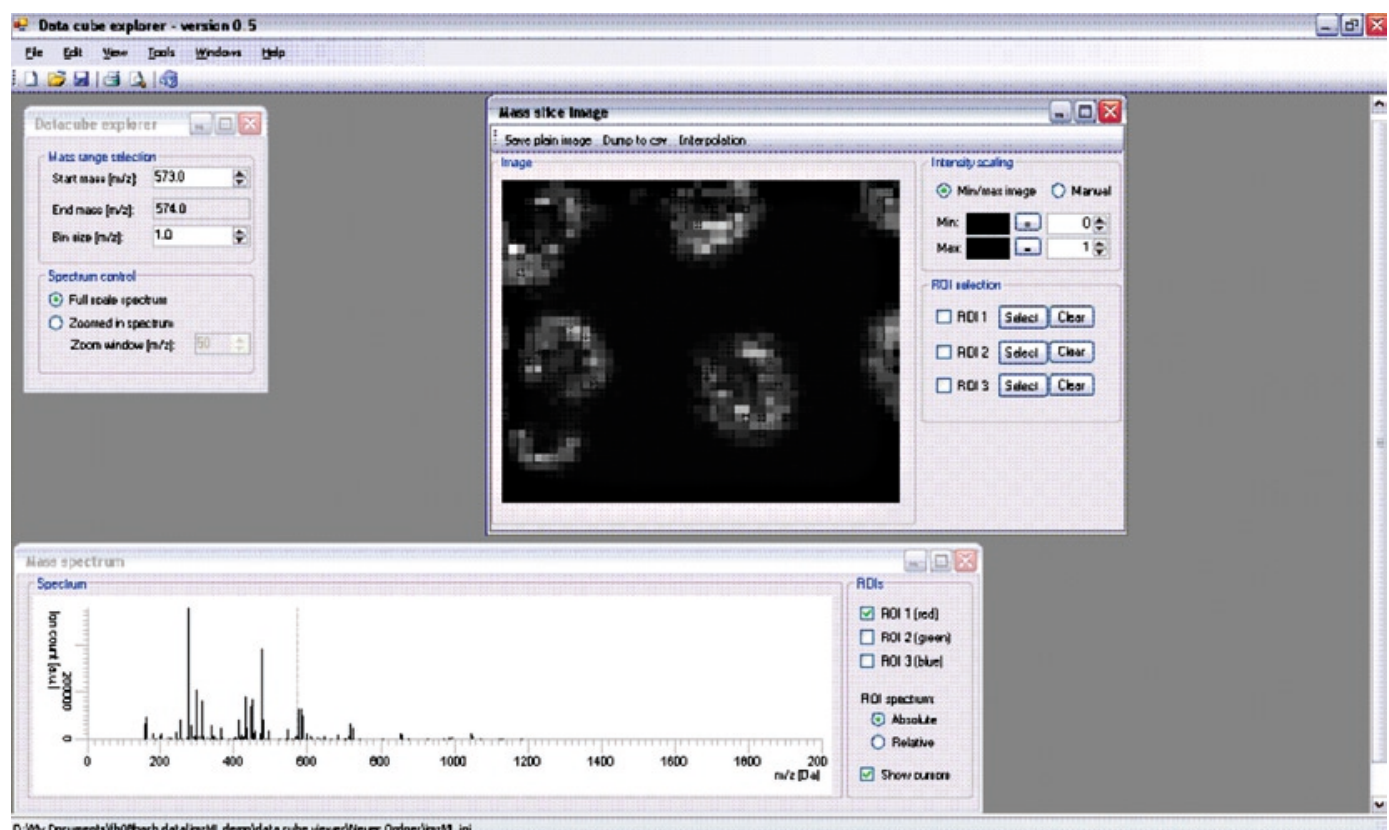

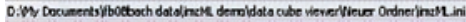

Fig. 10. Datacube Explorer (AMOLF).

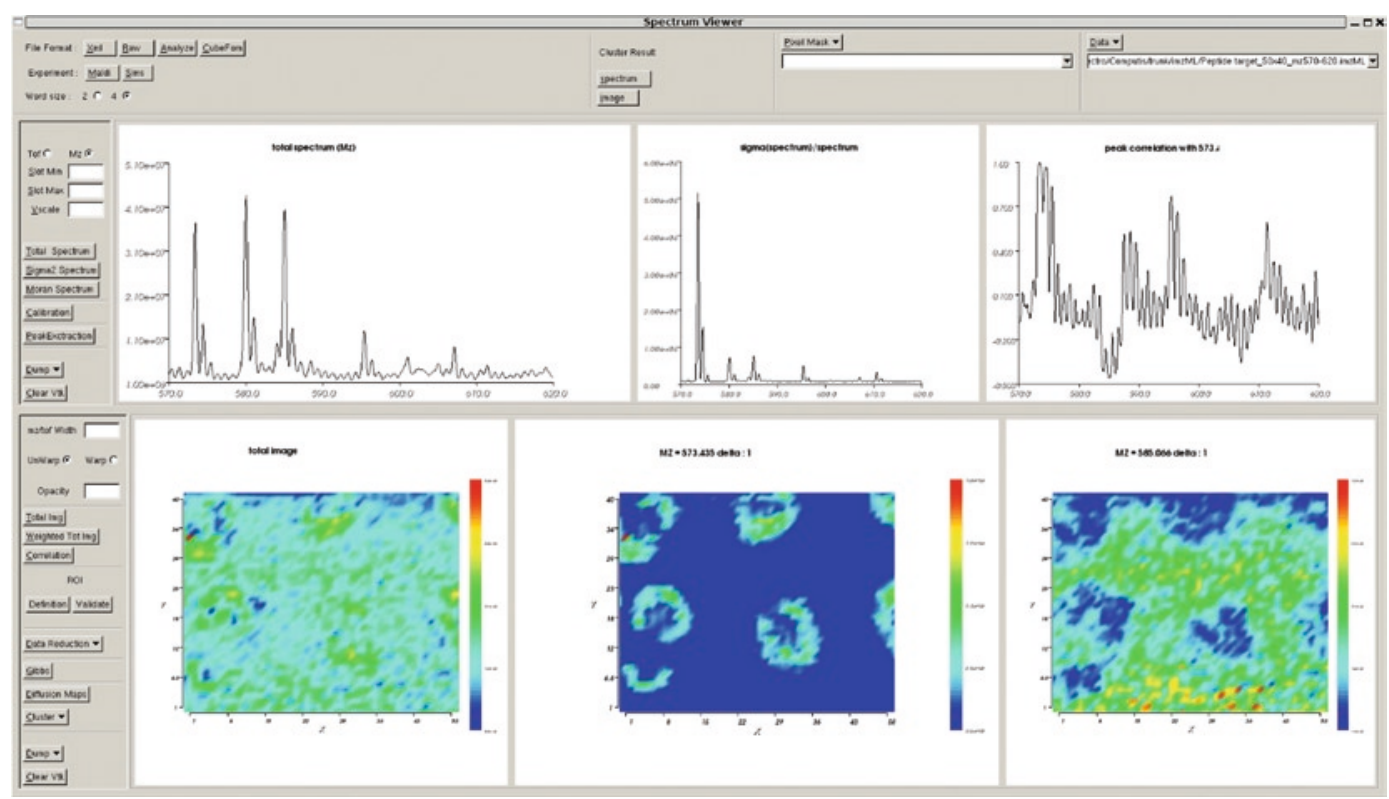

Fig. 11. fxSpectViewer (CEA). 


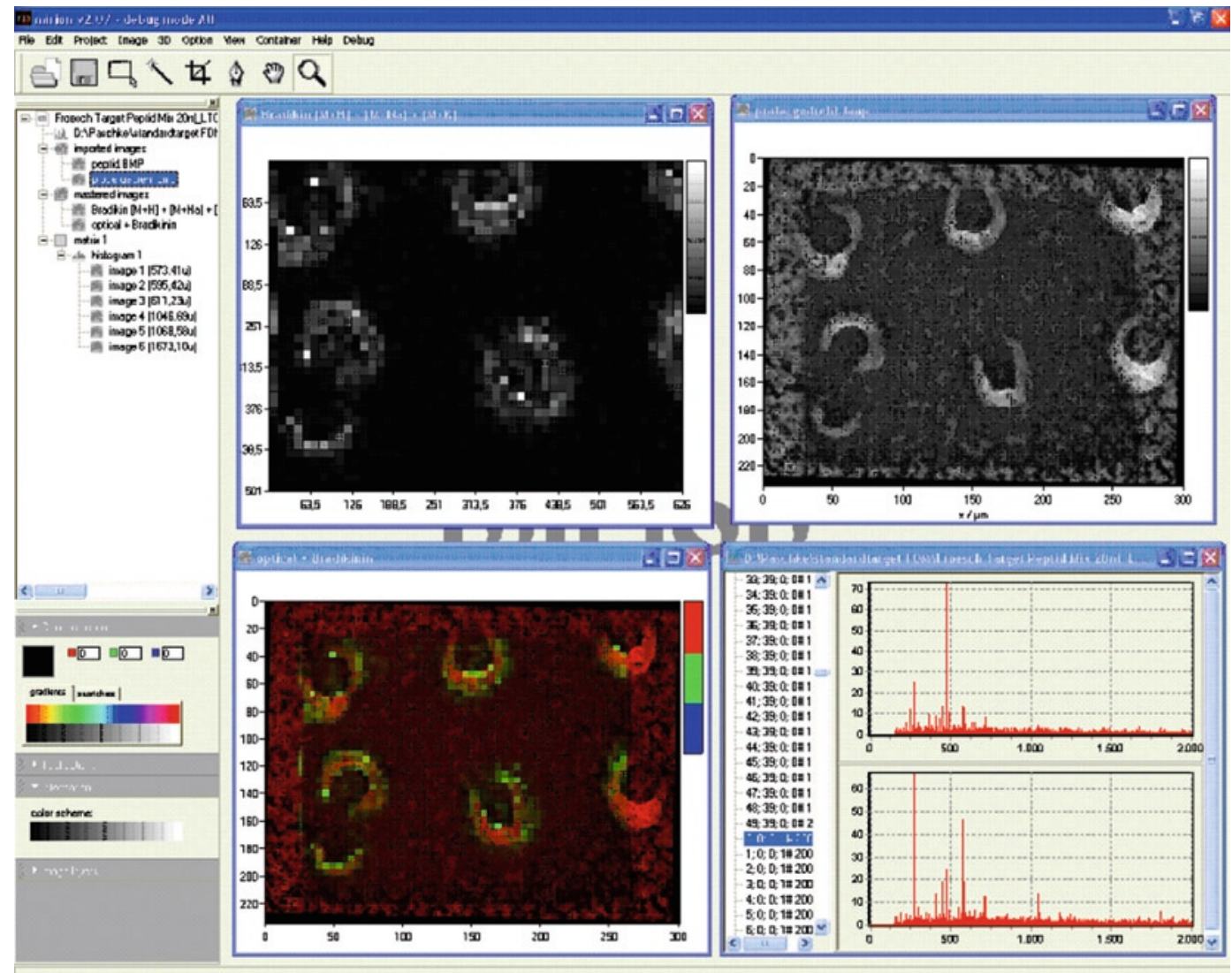

Fig. 12. Mirion (Justus Liebig University).

\subsection{Converters}

includes automatic segmentation of images. This software runs under Linux and Windows.

Mirion by JLU was especially developed for analyzing high mass resolution images (Fig. 12). It allows a bin width of 0.001 mass units. This is necessary to take full advantage of the highly accurate mass data from FTMS instruments. It also allows overlaying different MS images as well as optical images. Individual mass spectra are directly accessible from the image.

Several converters for imzML are currently developed and some are already available on the imzML website (http://www.imzML. org). An example and general considerations for the conversion of proprietary data to the imzML format is discussed in the following. The example shows a software that converts LTQ-based *.RAW files (proprietary format of Thermo Scientific GmbH, Bremen).

The "Conversion" tab contains details about the input RAW file. The "Imaging" tab (Fig. 13) includes the information that is essential to generate a valid imzML file - and therefore an unambiguous image. "Binary Mode" determines in which way the data 


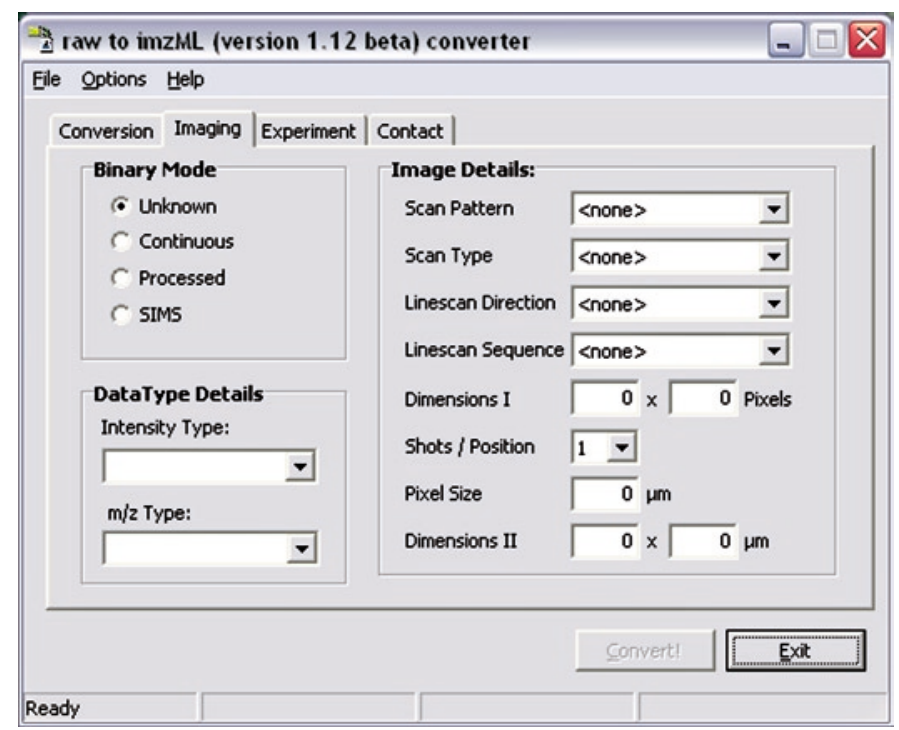

Fig. 13. imzML converter: User interface for imaging details.

is stored in the binary file. This option can have significant influence on the size of the resulting imzML file (see also Fig. 8). A discussion on which binary mode to use (processed or continuous) can be found in Note 3 .

The second property that influences the file size is the data type which is used to store the values of the $\mathrm{m} / \mathrm{z}$ and intensity arrays. This information is included in the "Data Type Details." Considerations for choosing the appropriate parameters are given in Note 4.

The original file might not contain all necessary information to generate a valid imzML file. This implies that the user has to add these details manually, for example, the entries on the right side ("Image Details"). The first four properties specify the characteristics of the scanning procedure which was used when the image was acquired (see Subheading "Scan Pattern").

Further information to be put in manually is included in the "Experiment" tab (Fig. 14). The parameters on the left describe the laser which was used in the MALDI imaging experiment. On the right side, the properties of the sample are listed. These parameters can be included, but they are not required to create a valid imzML file

When programming such a converter it is important to keep in mind that redundant information should be avoided if possible (for the sake of small data files). Some information might be stored redundantly in XML part of imzML. If for example data is acquired within an imaging experiment, all the measurements are 


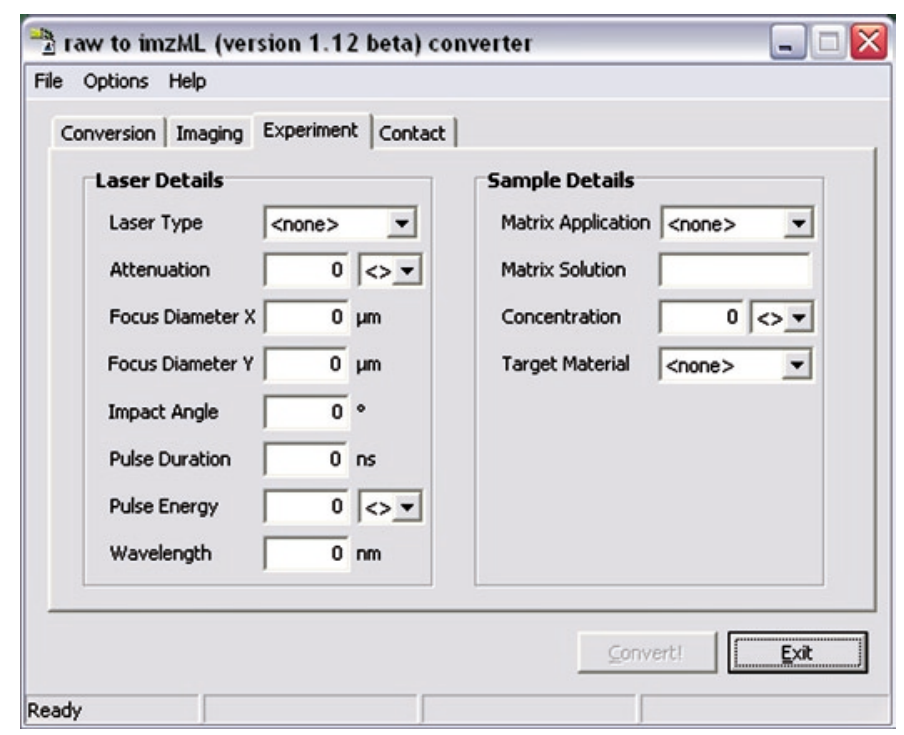

Fig. 14. imzML converter: User interface for experimental details.

usually acquired with the same instrumental settings and measurement conditions. Therefore it is sufficient to store this information only once per experiment and not for each spectrum separately. This redundant kind of information can nicely be merged into referenceable parameter groups (8). The file size can be reduced further by omitting $\mathrm{CV}$ param values that can be retrieved from the mass spectral data (e.g., base peak intensity).

\section{Notes}

1. Why not use mzML?

First of all mzML was not available at the beginning of the COMPUTIS project (2006). At later stages we evaluated mzML with respect to imaging MS data. The main concern was the file size of converted data sets. Storing the MS data in a separate binary file is crucial for handling the very large imaging MS data sets. After it became clear that our main requirement (a separate binary file) was not possible in mzML, we decided to continue with our own format. However, we decided to store our metadata in the mzML format in order to be able to easily convert between the two formats. During the last years we stayed in contact with HUPO-PSI at various occasions. The result of these discussions was that we call our format imzML (for imaging mzML) and that it will exist in parallel to mzML for specific use cases 
(e.g., large data sets). The structure of the XML metadata file in imzML will remain compatible with mzML. The imaging specific CV parameters will be kept in a separate imagingMS obo file. A number of entries from this file (which were of general importance) have already been included in the PSI-MS OBO file.

imzML files can easily be converted to mzML files (with the consequence of increased file size and limited metadata) in order to use mzML-based tools.

2. Byte order of UUID

Binary data can be stored in "little endian" or "big endian" (net standard) byte order. Mass spectral data in imzML binary files is stored in little endian byte order. The universally unique identifier (UUID), however, is stored in big endian according to the RFC 4122 specifications (cf.).

Intel processors and clones use little endian, therefore integers in the computer memory are also little endian numbers. Depending on the programming language which is used, byte order may be automatically "corrected" when reading big endian UUIDs. But some widely used programming languages have no such automatic correction. So the programmer has to take care of this.

In the imzML binary file the first 16 bytes are the binary representation of the UUID. A hexadecimal viewer can be used to examine these bytes, for example: 5233 F9 E6 09 B9 4A $00 \mathrm{AB} 01 \mathrm{AF} 5 \mathrm{D} \mathrm{F} 4 \mathrm{BE} 3815$.

In the corresponding imzML file the textual version of the UUID which consists of 5 blocks delimited by a “-"-char is: \{5233F9E6-09B9-4A00-AB01-AF5DF4BE3815\}. Be aware that in a correct implementation, both representations should have the same sequence of hexadecimal numerals.

When implementing imzML on a Microsoft operating system, one will usually use the Microsoft implementation of UUID: GUID (general unique identifier). Its memory representation is defined by:

TGUID = structure

Datal : 4 byte unsigned integer;

Data2 : 2 byte unsigned integer;

Data3 : 2 byte unsigned integer;

Data4 : array of 8 bytes;

end structure;

In this structure, Datal, Data2, and Data3 are numbers and therefore subject to byte order, whereas Data4 is just an array of 8 bytes (and thus independent of byte order). That means when dumping this memory representation on a little 
endian computer into a binary file Datal, Data2 and Data3 are inverted whereas Data4 is not inverted.

3. Continuous or processed format

The binary mode (continuous or processed), which is used in the binary file has significant influence on the overall size of imzML files and is therefore an important parameter. Data with a continuous mass axis is often generated by time-offlight instruments. Discontinuous data can be the result of data processing. In some cases the appropriate (most effective) data format can vary for one instrument depending on the settings and mode of operation. For example, LTQ-based instruments can generate profile or continuous data in the linear ion trap mode. Measurements performed in the centroid mode always have to be saved as "processed" due to the discontinuous mass axis. When the acquisition is set to profile mode the data format depends on the mass analyzer used for the acquisition. Data from Fourier transform analyzers (ICR or Orbitrap) is always modified before storage and thus has to be stored as "processed" (because of on-the-fly dataprocessing). If the linear ion trap analyzer is used for detection the data can be saved in continuous mode.

It is possible to convert continuous into processed data. The $\mathrm{m} / \mathrm{z}$ values of the first spectrum simply have to be saved for every spectrum. After conversion, all advantages of the processed mode are usable: for example, skipping zero intensity values.

A special case is the storage of data acquired by secondary ion mass spectrometry (SIMS). This data is typically stored in an "event-based" format due to the much lower number of ions detected in this ionization mode. A data point consists of three values: $\mathrm{x}$, $\mathrm{y}$ coordinates, and $\mathrm{m} / \mathrm{z}$ (or time-of-flight) value. This data has to be converted in order be stored in the binary file. For each pixel the events are sorted by increasing $\mathrm{m} / \mathrm{z}$ values (after conversion from time-of-flight, if necessary). These data have to be binned in order to be stored as mass spectra: events within a defined mass bin (e.g., 1 u) are summed up. The binned data can be stored in the binary file of the imzML format (usually in the "processed mode").

It has to be stressed again that the most efficient data format strongly depends on the type of data and has to be evaluated for each set of experiments.

4. How to choose the appropriate binary data type?

As already mentioned above the size of the binary file is dependent on the applied data. Choosing an unsuitable data type either results in loss of information or unnecessarily large data files. Some considerations on deciding which data type 
to use for $\mathrm{m} / \mathrm{z}$ and intensity values, respectively, are given below. If the data of a mass spectrometry imaging experiment was acquired by a very accurate mass spectrometer (e.g., FT-ICR), the accuracy of the measured $\mathbf{m} / \mathbf{z}$ values (up to eight significant digits) should be taken into account by choosing the "64 bit float" (double precision) data type (up to 15 significant digits). Storing the data in "32 bit float" (single precision) would only allow seven significant digits, resulting in a loss of precision.

Intensity values of acquired spectra can be of integer or floating point data type. This depends on the way the ions are detected and on the digitizer of the used mass spectrometer. The best data type for integer values can be estimated by taking a look at the maximum intensity value of all spectra. If for example, the maximum intensity value is 125 then a data type of 8-bit integer will be sufficient to store these values. The usage of a data type with a range too small for all the values of a mass spectrometry imaging experiment will result in a loss of information. The same applies for the usage of an integer data type for floating point values, because the decimals of the numbers will be cut off by rounding.

\section{References}

1. McDonnell LA, Heeren RMA (2007) Imaging mass spectrometry. Mass Spectrom Rev 26:606-643

2. EU Project COMPUTIS (Accessed at http:// www.computis.org)

3. Digital imaging and communications in medicine - DICOM. (Accessed at http:// medical.nema.org)

4. Deutsch E (2008) mzML: a single, unifying data format for mass spectrometer output. Proteomics 8:2776-2777

5. Hermjakob H (2006) The HUPO proteomics standards initiative - Overcoming the fragmentation of proteomics data. Proteomics 6(Suppl 2):34-38

6. Orchard S, Hermjakob H, Taylor CF et al (2005) Second Proteomics Standards Initiative Spring Workshop. Expert Rev Proteomics 2:287-289

7. Biomap. (Accessed at http://maldi-msi.org/ index.php?option $=$ com_content $\&$ task $=$ view \&id $=14 \&$ Itemid $=39$ )

8. HUPO PSI mzML documentation version 1.1. (Accessed at http://psidev.info/index. php?q=node $/ 257$ )
9. RFC 4122 - A Universally Unique IDentifier (UUID) URN Namespace. (Accessed at http://tools.ietf.org/html/rfc4l22)

10. Smith B, Ashburner M, Rosse C et al (2007) The OBO foundry: coordinated evolution of ontologies to support biomedical data integration. Nat Biotechnol 25: $1251-1255$

11. Cote RG, Jones P, Martens L, Apweiler R, Hermjakob H (2008) The ontology lookup service: more data and better tools for controlled vocabulary queries. Nucleic Acids Res 36:372-376

12. Ontology of Mass Spectrometry Imaging. (Accessed at http://www.maldi-msi.org/ index.php?option $=$ com_content\&view $=$ articl e\&id $=187 \&$ Itemid $=67$ )

13. PSI MS obo. (Accessed at http://psidev.cvs. sourceforge.net/checkout/psidev/psi/psi$\mathrm{ms} / \mathrm{mzML} /$ controlledVocabulary/psi-ms. obo)

14. OECD (1995) The application of the principles of GLP to computerised systems 